OPEN ACCESS

Edited by:

Hanting Zhang

West Virginia University, USA

Reviewed by:

Shijun Yan,

University of Kansas, USA

Min-Yu Sun,

Washington University in St. Louis,

USA

*Correspondence: Wensheng Zhang zws@bnu.edu.cn

Specialty section:

This article was submitted to

Neuropharmacology,

a section of the journal

Frontiers in Pharmacology

Received: 16 December 2016 Accepted: 13 February 2017

Published: 27 February 2017

Citation:

Zhang S, Zhu D, Li H, Zhang H,

Feng $C$ and Zhang W (2017) Analyses

of mRNA Profiling through RNA

Sequencing on a SAMP8 Mouse

Model in Response to Ginsenoside

$R g 1$ and Rb1 Treatment.

Front. Pharmacol. 8:88.

doi: 10.3389/fphar.2017.00088

\section{Analyses of mRNA Profiling through RNA Sequencing on a SAMP8 Mouse Model in Response to Ginsenoside Rg1 and Rb1 Treatment}

\author{
Shuai Zhang 1,2,3, Dina Zhu ${ }^{1,2,3}$, Hong $\mathrm{Li}^{1,2,3}$, Haijing Zhang ${ }^{1,2,3}$, Chengqiang Feng ${ }^{1,2,3}$ and \\ Wensheng Zhang 1,2,3, 4*
}

${ }^{1}$ Beijing Area Major Laboratory of Protection and Utilization of Traditional Chinese Medicine, Beijing Normal University, Beijing, China, ${ }^{2}$ Engineering Research Center of Natural Medicine, Ministry of Education, Beijing Normal University, Beijing, China, ${ }^{3}$ Department of Chinese Medicine, College of Resources Science Technology, Beijing Normal University, Beijing, China, ${ }^{4}$ National and Local United Engineering Research Center for Sanqi Resources Protection and Utilization Technology, Kunming, China

Ginsenoside Rg1 and Rb1 are the major ingredients in two medicines called QiShengLi (Z20027165) and QiShengJing (Z20027164) approved by China. These ingredients are believed to mitigate forgetfulness. Numerous studies have confirmed that GRg1 and GRb1 offer protection against Alzheimer's disease (AD), and our morris water maze (MWM) experiment also indicated that GRg1 and GRb1 may attenuate memory deficits in the 7-month-old SAMP8 mice; however, comprehensive understanding of their roles in $A D$ remains limited. This study systematically explored the mechanism at the genome level of the anti-AD effects of GRg1 and GRb1 in a senescence-accelerated mouse prone 8 (SAMP8) model through deep RNA sequencing. A total of 74,885 mRNA transcripts were obtained. Expression analysis showed that 1,780 mRNA transcripts were differentially expressed in SAMP8 mice compared with the SAMP8+GRg1 mice. Moreover, 1,066 significantly dysregulated mRNA transcripts were identified between SAMP8 and SAMP8+GRb1 mice. Analyses according to gene ontology and the Kyoto Encyclopedia of Genes and Genomes revealed that oral administration of GRg1 and GRb1 improved the learning performance of the SAMP8 mouse model from various aspects, such as nervous system development and mitogen-activated protein kinase signaling pathway. The most probable AD-related transcriptional responses after medication were predicted and discussed in detail. This study is the first to provide a systematic dissection of mRNA profiling in SAMP8 mouse brain in response to GRg1 and GRb1 treatment. We explained their efficacy thoroughly from the source (gene-level explanation). The findings serve as a theoretical basis for the exploration of GRg1 and GRb1 as functional drugs with anti-AD activity.

Keywords: Ginsenoside Rg1, Ginsenoside Rb1, Alzheimer's disease, SAMP8, gene-level, RNA sequencing

\section{INTRODUCTION}

Alzheimer's disease $(\mathrm{AD})$ is a progressive amnesic disorder characterized by age-dependent memory loss and impairment of cognitive functions (Yaari and Corey, 2007; Roy et al., 2016). According to reports, two types of AD exist. Sporadic AD accounts for 90-95\% of all AD cases, and the familial form of $\mathrm{AD}$ accounts for 5-10\% (Bhat, 2010; Babusikova et al., 2011). Current statistics 
indicate that a person in America develops AD every $67 \mathrm{~s}$. By 2050, a new case of $\mathrm{AD}$ is expected to develop every $33 \mathrm{~s}$, and nearly 1 million new cases per year is expected to arise (Alzheimer's Association, 2015). AD is becoming a major public health problem, and discovering effective drugs for the treatment, relief, or prevention of $\mathrm{AD}$ has become increasingly urgent.

Chinese medicinal herbs exert certain effects on the treatment of neurodegenerative diseases (Howes et al., 2003). Panax notoginseng, the root of Panax notoginseng (Burk.) F. H. Chen, is a well-known traditional Chinese herbal medicine with a long history of usage in East Asian countries (Yan et al., 2014). Panax notoginseng contains more than 30 different saponins called Panax notoginsenosidum (PNS). Ginsenosides Rg1 (GRg1) and Rb1 (GRb1) are the major pharmacologically active ingredients of Panax notoginseng (Du et al., 2003). As eminent members of PNS, GRg1, and GRb1 are believed to mediate the complex pathological mechanisms of $\mathrm{AD}$. Zhao et al. reported that GRg1 suppresses the signaling transduction pathway of TLR3 and TLR4 and decreases the inflammation factors induced by $A \beta 25-35$ in NG10815 cells (Zhao et al., 2014). Scientific evidence shows that GRb1 reverses the changes in several direct or indirect neuroinflammation markers of $\mathrm{AD}$ produced by ventricle injection of A $\beta$ 1-42 (Wang et al., 2011). Li et al. concluded that GRg1 treatment affects all three metabolic pathways, and GRb1 treatment affects lecithin and amino acid but not sphingolipid metabolism in AD mice (Li et al., 2015). These studies provided several clues but not detailed insights and comprehensive assessments.

In the present study, the mRNA profiles of the brain of GRg1- and GRb1-treated senescence-accelerated mouse prone 8 (SAMP8) models at 7 months of age were explored through RNA sequencing. The SAMP8 strain is a naturally derived model that demonstrates cognitive, behavioral, and neuropathological alterations similar to those observed in aged humans; it is a plausible model for exploring the complexity of $\mathrm{AD}$ (Butterfield and Poon, 2005; Cheng et al., 2014; Kang et al., 2014). This research systematically provided a molecular basis for the therapeutic benefits of GRg1 and GRb1 in AD treatment. Thus, GRg1 and GRb1 may be potential therapeutic reagents for halting or preventing $\mathrm{AD}$ progression.

\section{MATERIALS AND METHODS}

\section{Preparation of GRg1 and GRb1}

GRg1 $\left(\mathrm{C}_{42} \mathrm{H}_{72} \mathrm{O}_{14}\right.$, molecular weight $=801.01$, Figure 1) and GRb1 $\left(\mathrm{C}_{54} \mathrm{H}_{92} \mathrm{O}_{23}\right.$, molecular weight $=1,109.29$, Figure 1) with purity $>98 \%$ were purchased from the Chinese National Institute for the Control of Pharmaceutical and Biological Products (Beijing, China). GRg1 and GRb1 were dissolved in sterile distilled water before use.

\section{Animals and Grouping}

Three-month-old male SAMP8 mice $(n=45)$ were purchased from Beijing WTLH Biotechnology Co., Ltd. Each mouse was housed in a cage in standard specific pathogen-free conditions (24 $\pm 2{ }^{\circ} \mathrm{C}, 45-55 \%$ humidity, and $12 \mathrm{~h}$ light/dark cycle). The mice were allowed free access to water and food. The SAMP8 mice were randomly divided into three groups $(n=15$ mice per group), which received GRg1 (15 mg/kg/d), GRb1 (15 mg/kg/d), and the vehicle (sterile distilled water) via oral gavage. After 4 months of GRg1 and GRb1 administration, three animals were randomly selected from each group and anesthetized to collect the cerebral cortex. The tissues were immediately frozen in liquid nitrogen for RNA sequencing. Eight animals in each group were also randomly selected for the morris water maze (MWM) test.

All of the animal procedures were approved by the Institutional Animal Use and Care Committee of Beijing Normal University and adhered to the "Guide for the care and use of laboratory animals” (Clark et al., 1997).

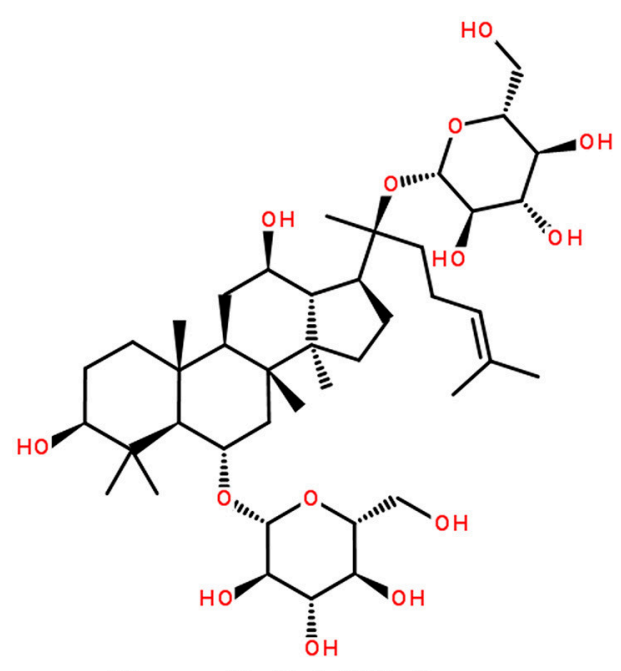

Ginsenoside Rg1 (GRg1)

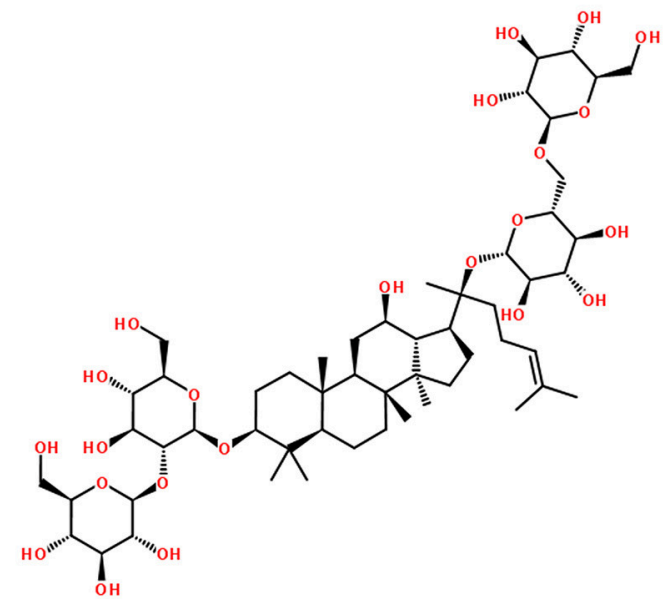

Ginsenoside Rb1 (GRb1)

FIGURE 1 | Structures of GRg1 and GRb1. 


\section{MWM Test}

The spatial learning and memory of the mice were evaluated through the MWM test (D'Hooge and De Deyn, 2001; Vorhees and Williams, 2006). Briefly, in the hidden platform test (days 1-5), a platform was placed at the center of a suppositive quadrant. The mice were subjected to two trials per day for five consecutive days. During each trial, the mice were placed in the maze at four different assigned points and allowed to swim for $90 \mathrm{~s}$. The escape latency was recorded by a software upon mounting the platform. When a mouse failed to reach the platform within $90 \mathrm{~s}$, it was guided to the platform, and the escape latency was recorded as $90 \mathrm{~s}$. In both situations, the mice were allowed to rest on the platform for $15 \mathrm{~s}$ and subsequently placed in the home cage. The platform was removed in the spatial probe test (day 6). The mice were released from the opposite quadrant and allowed to swim freely for $60 \mathrm{~s}$. All experiments were conducted at approximately the same time daily.

\section{RNA Extraction and Qualification}

TRIzol reagent (Invitrogen, Carlsbad, CA, USA) was used to isolate the total RNA of each sample. The purity of RNA was checked with a NanoPhotometer ${ }^{\circledR}$ spectrophotometer (IMPLEN, Westlake Village, CA, USA). The concentration of RNA was tested with Qubit ${ }^{\circledR}$ RNA Assay Kit in Qubit 2.0 Flurometer (Life Technologies, CA, USA). In addition, RNA integrity was assessed with RNA Nano 6000 Assay Kit in the Bioanalyzer 2100 system (Agilent Technologies, Santa Clara, CA, USA). The samples with RNA integrity number scores higher than eight were used in this study.

\section{Library Preparation and Sequencing}

Nine cDNA libraries were constructed, i.e., three for the SAMP8+GRg1 mice, three for the SAMP8+GRb1 mice, and three for the SAMP8+vehicle mice. We utilized $3 \mu \mathrm{g}$ of RNA per sample as the input material for RNA sample preparation. First, rRNA-depleted RNA was fragmented with NEBNext first strand synthesis reaction buffer (5X). Second, a random hexamer primer and $\mathrm{M}-\mathrm{MuLV}$ reverse transcriptase $(\mathrm{RNaseH}-)$ were used to synthesize the first-strand cDNA. Second-strand cDNA was synthesized subsequently with DNA polymerase I and RNase $H$. Third, the purified second-strand cDNA was ligated with NEBNext adaptor after adenylation of the 3' ends of the DNA fragment, and 150-200 bp cDNA fragments were isolated. Then, the cDNA libraries were enriched through PCR amplification with Phusion high-fidelity DNA polymerase, universal PCR primers, and index (X) primer. The Agilent bioanalyzer 2100 system was used to assess library quality. The libraries were sequenced at the Novogene Bioinformatics Institute (Beijing, China) on an Illumina HiSeq 4000 platform, and 150 bp pairedend reads were generated after clustering of the index-coded samples. Clustering was performed in a cBot cluster generation system using the TruSeq PE Cluster Kit v3-cBot-HS (Illumina).

\section{Transcriptome Assembly}

Clean data were obtained after filtering out reads with adaptors and poly- $\mathrm{N}>10 \%$ and low-quality reads from raw data through in-house perl scripts. The Q20, Q30, and GC contents of the clean reads were calculated. All downstream analyses were based on the good-quality clean reads. Paired-end clean reads were mapped to the mouse genome sequence (ftp://ftp.ensembl.org/pub/release81/gtf/mus_musculus/) with TopHat v2.0.9 (Trapnell et al., 2009; Kim et al., 2013). Cufflinks v2.1.1 was used to assemble the mapped reads of each sample (Trapnell et al., 2010).

\section{Expression Analysis}

The protein-coding gene expression levels in each sample were estimated according to fragments per kilo-base of exon per million fragments mapped (FPKMs) and assessed with Cufflinks v2.1.1 (Trapnell et al., 2010). Transcripts with a $P<0.05$ were considered differentially expressed.

\section{Quantitative Polymerase Chain Reaction (qPCR)}

The results of RNA sequencing were validated through qPCR. qPCR was performed with the LightCycler 480 real-time PCR system and SYBR Green PCR Master Mix (TaKaRa Biotechnology, Dalian, China). The specific quantitative primers for 8 transcripts (four from SAMP8 vs. SAMP8+GRg1 and four from SAMP8 vs. SAMP8+GRb1) are listed in Supplementary Table 1 . The $20 \mu \mathrm{L}$ reaction volume contained $0.5 \mu \mathrm{L}$ of each primer, $8 \mu \mathrm{L}$ of $\mathrm{H}_{2} \mathrm{O}, 1 \mu \mathrm{L}$ of cDNA, and $10 \mu \mathrm{L}$ of $2 \times$ RealMasterMix (TaKaRa Biotechnology). The conditions were $95^{\circ} \mathrm{C}$ for $2 \mathrm{~min}$ followed by 40 cycles $\left(95^{\circ} \mathrm{C}\right.$ for $20 \mathrm{~s}, 60^{\circ} \mathrm{C}$ for $30 \mathrm{~s}$, and $68^{\circ} \mathrm{C}$ for $30 \mathrm{~s}$ ). Each experiment was performed in triplicate.

\section{Gene ontology (GO) and Kyoto Encyclopedia of Genes and Genomes (KEGG) Analyses}

To understand the functional roles of the differentially expressed protein-coding genes, we used the GOseq $\mathrm{R}$ package to conduct an enrichment analysis (Young et al., 2010). GO terms with a $P<$ 0.05 were considered significantly enriched. In addition, KEGG enrichment analysis was performed with KOBAS software on the differentially expressed protein-coding genes (Mao et al., 2005) through a hypergeometric test. A hypergeometric $P<0.05$ was considered significant.

\section{Statistical Analysis}

Statistical analyses were performed with SPSS 20.0 software. All data were expressed as the means \pm standard error of the mean (SEM). $P<0.05$ were considered statistically significant. The escape latency in the MWM test was compared through two-way analysis of variance (ANOVA). One-way ANOVA was used to analyze the rest index in the MWM test. Student's $t$-test was used to compare the $\mathrm{qPCR}$ results.

\section{RESULTS}

\section{GRg1 and GRb1 Ameliorated Memory Impairment in 7-Month-Old SAMP8 Mice}

We performed the MWM test to evaluate the effect of GRg1 and GRb1 on the cognitive function of the SAMP8 mouse model. In the result, we introduced the MWM performance of the senescence-accelerated mouse resistant 1 (SAMR1) mouse model based on our previous study (Zhang et al., 2016). The SAMR1 
mouse model is widely used as a control strain (Ma et al., 2011). As shown in Figure 2A, the mean escape latency of SAMP8 + GRg1, SAMP8 + GRb1, and SAMR1 mice significantly decreased compared with that of SAMP8 + vehicle mice $(P<0.05)$. In the spatial probe test, the SAMP8 mice swam randomly in the tank without knowing the target location, whereas the GRg1and GRb1-treated SAMP8 mice and SAMR1 mice searched preferentially for the target quadrant and showed improved memory (Figure 2B). In addition, the number of crossings and the time spent in the target quadrant increased significantly in the GRg1- and GRb1-treated SAMP8 mice and SAMR1 mice compared with the SAMP8 mice (Figures 2C,D). No significant difference was observed in the swimming speed of all the groups $(P>0.05$, Figure $2 \mathrm{E})$, suggesting that the memory impairments of SAMP8 mice were not caused by motor and visual dysfunctions. Evidently, administration of GRg1 and GRb1 for 4 months significantly attenuated the memory deficit of 7-month-old SAMP8 mice.

\section{Overview of RNA Sequencing}

A total of $800,400,106$ raw reads $(251,783,324$ for SAMP8, $277,307,450$ for SAMP8+GRg1, and 271,309,332 for SAMP8+GRb1) were generated. After discarding the reads with adapters, poly- $\mathrm{N}>10 \%$, and any other possible contaminants, $784,513,910$ clean reads $(244,770,474$ for SAMP8, 272,566,802 for SAMP8+GRg1, and 267,176,634 for SAMP8+ GRb1) were obtained. The clean reads were mapped to the mouse reference genome (ftp://ftp.Ensemble.org/pub/release-83/gtf/ mus_musculus/), and the mapping rates were approximately $70.72,71.41$, and $70.06 \%$ for the SAMP8, SAMP8+GRg1, and SAMP8+GRb1 mice, respectively. The cufflinks results indicated that 102,250 transcripts were assembled. A total of 74,885 protein-coding transcripts were identified. These mRNAs were used for subsequent analysis.

\section{Differential Expression Analysis: SAMP8 vs. SAMP8+GRg1 and SAMP8 vs. SAMP8+GRb1}

The mRNA expression levels and transcripts were estimated with FPKMs. A total of 1,780 mRNA transcripts, including 768 upregulated and 1,012 downregulated transcripts $(p<0.05)$, were differentially expressed in SAMP8+GRg1 mice relative to SAMP8 mice (Supplementary Table 2). Meanwhile, 1,066 significantly dysregulated mRNA transcripts were identified between SAMP8 and SAMP8+GRb1 mice (Supplementary Table 3); 381 were upregulated in the SAMP8 mice, whereas 685 were downregulated in SAMP8+GRb1 mice $(p<0.05)$. Cluster analysis of differentially expressed mRNAs was conducted with heat maps (Figures 3A,B).

\section{Quantitative Assessment}

Eight differentially expressed mRNA transcripts (four from SAMP8 vs. SAMP8+GRg1 and four from SAMP8 vs. SAMP8+GRb1) were randomly selected to validate the accuracy of RNA sequencing through qPCR. All the selected mRNA transcripts were detected and exhibited significant differential expressions (Figure 4). These results are consistent with RNA sequencing data.

\section{Functional Annotation}

First, GO and KEGG analyses were performed on 1,780 significantly dysregulated mRNAs in SAMP8 vs. SAMP8+GRg1. We derived 1,575 highly enriched GO terms (Supplementary Table 4, $P<0.05$ ) and 21 significantly enriched

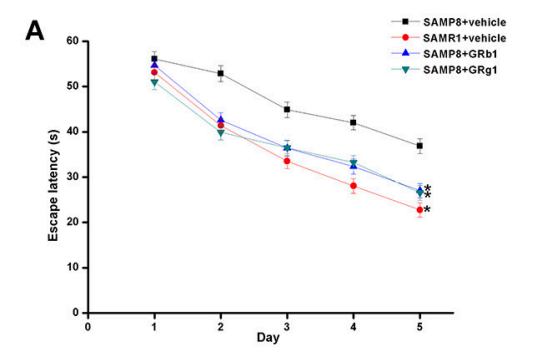

C

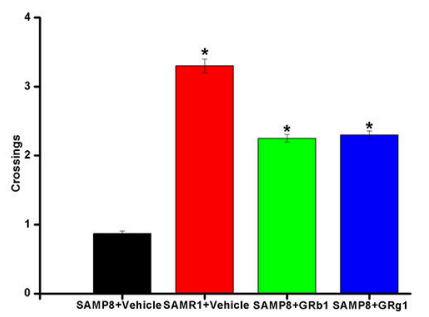

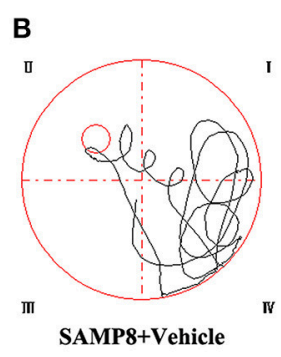

D

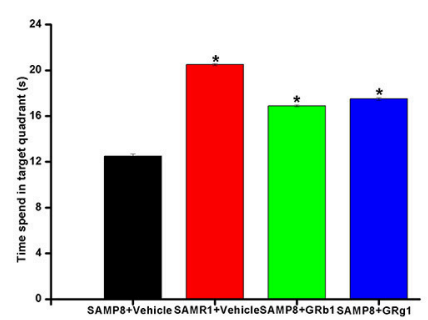

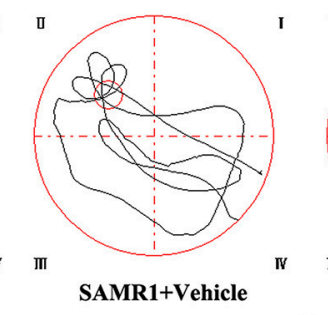

E
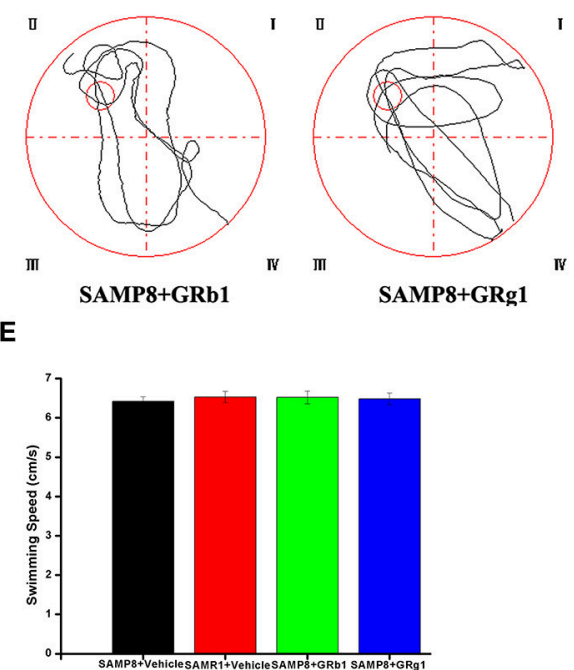

FIGURE 2 | GRg1 and GRb1 administration ameliorated memory deficit in SAMP8 mice. Morris water maze tests were conducted on mice with or without GRg1 and GRb1 (15 mg/kg/d, 4 months) treatment. Eight animals in each group. (A) Mean escape latency in the hidden platform test. (B) Swimming paths in the spatial probe test. (C) Number of crossings in the spatial probe test. (D) Time spent in the target quadrant during the spatial probe test. (E) Average swimming speeds of mice in the visible-platform test. ${ }^{*} p<0.05$. 
A
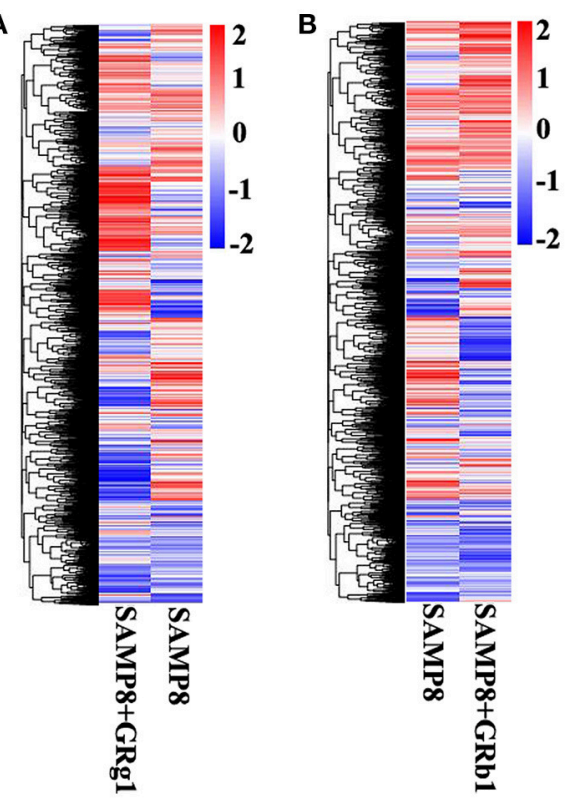

FIGURE 3 | Cluster analysis by heat map. (A) Cluster analysis of differentially expressed mRNAs in SAMP8 and SAMP8+GRg1 mice. (B) Cluster analysis of differentially expressed mRNAs in SAMP8 and SAMP8+GRb1 mice. The red markings indicate an increased expression, and the blue markings indicate a decreased expression.

pathways (Supplementary Table 5, $P<0.05$ ). We observed several $\mathrm{AD}$-associated pathways and terms, such as protein processing in the endoplasmic reticulum, FoxO signaling, $\mathrm{PI} 3 \mathrm{~K}-$ Akt signaling, and mitogen-activated protein kinase (MAPK) signaling pathways, and several GO terms (e.g., GO: 0031175, GO: 0007399, GO: 0045686, GO: 0010977, and GO: 0043409). Overall, GRg1 affected AD from different angles.

GO and pathway enrichment analyses were also executed to explore the functions of 1,066 significantly dysregulated mRNAs in SAMP8 vs. SAMP8+GRb1. According to the GO survey, 1,368 GO terms were significantly enriched (Supplementary Table 6, $P<0.05)$. Several terms, including GO: 0000188, GO: 0007420, and GO: 0007416, were closely related to AD. Eighteen significantly enriched KEGG pathways (Supplementary Table 7, $P<0.05)$ were detected. Several of these pathways, including the MAPK signaling pathway, Notch signaling pathway, and cholinergic synapse, were associated with $\mathrm{AD}$. Overall, the pathological process of $\mathrm{AD}$ may be regulated by GRb1 from different aspects.

\section{Comprehensive Analysis}

In our previous study (Zhang et al., 2016), we identified 2,505 significantly dysregulated mRNA transcripts between SAMP8 and SAMR1 mice. Thus, to further understand the potential protective effects of GRg1 and GRb1 on AD, we set two limiting factors. One was that the gene should be significantly and differentially expressed between SAMP8 vs. SAMP8+GRg1 or SAMP8 vs. SAMP8+GRb1 mice. At the same time, the change in the expression after administrating GRg1 or GRb1 must lean toward the normal group (SAMR1). The other requirement was that the selected gene should be associated with AD. According to these requirements, the fulfilled genes were detected and are listed in Tables 1, 2. For example, Mapk11 is an enzyme encoded by the Mapk11 gene (Stein et al., 1997). This kinase is closely related to p38 MAP kinase and is involved in the development of $\mathrm{AD}$. We found that the expression of Mapk11 in the SAMP8+GRb1 and SAMR1 mice was significantly lower than that in the SAMP8 mice. In addition, an evident reduction was observed in the SAMP8+GRg1 mice, but this reduction was not significant. In short, we predict that GRg1 and GRb1 may regulate the expression of the abovementioned genes and are possibly involved in the adjustment of AD.

\section{DISCUSSION}

The first detailed record of the medicinal use of Panax notoginseng was indicated in the Compendium of Materia Medica, which was written by Li Shizhen and published in A.D. 1596 (Chinese Ming Dynasty) (Zhang and Fang, 2004). PNS, the active ingredient extracted from the root of Panax notoginseng (Burk.) F.H. Chen (Araliaceae), has been extensively used to treat various diseases. Li et al. reported that PNS injections are widely applied in clinical situations in China (Li et al., 2009). GRg1 and GRb1, as known members of PNS, attract great attention, especially in reducing the risk of neurodegenerative diseases, such as AD (Wang et al., 2011; Kim et al., 2014; Zhao et al., 2014; Li et al., 2015). However, we believe that staying at this level is far from enough. RNA sequencing (Ozsolak and Milos, 2011), a modern technology, allows for the investigation of the AD-resistant effects of GRg1 and GRb1 in unprecedented detail. In this study, the 7-month-old SAMP8 mice developed severe deficits in learning and memory, and the MWM experiment confirmed this result. SAMP8 mice at this age are relatively "old" in their average life span of 12 months. Meanwhile, the SAMP8 strain is a naturally derived model and shares cognitive, behavioral, and neuropathological alterations observed in aged humans; it can be considered an appropriate animal model to understand the pathogenic mechanisms of $\mathrm{AD}$, especially sporadic AD (Cheng et al., 2014; Kang et al., 2014). However, the transgenic mouse models of $\mathrm{AD}$, such as $3 \mathrm{xTg}-\mathrm{AD}$, may simply represent the uncommon familial AD. The primary goal of this research was to systematically analyze the mRNA profiles of the brain of SAMP8 mice after 4 months of GRg1 and GRb1 administration through RNA sequencing. The results indicate that GRg1 and GRb1 can help restrict the development and progression of AD.

First, we identified a total of 74,885 mRNAs in SAMP8, SAMP8+GRg1, and SAMP8+GRb1 mice brains by using the Illumina HiSeq 4000 platform. Then, we compared the mRNA expressions of the SAMP8 and SAMP8+GRg1 mice. We also measured the differential mRNA expression between SAMP8 and SAMP8+GRb1 mice. A total of 1,780 dysregulated mRNA transcripts transcripted from 1,714 genes were detected in the SAMP8 vs. SAMP8+GRg1 group. Moreover, 1,066 differentially expressed mRNA transcripts corresponded to the 1,025 genes 


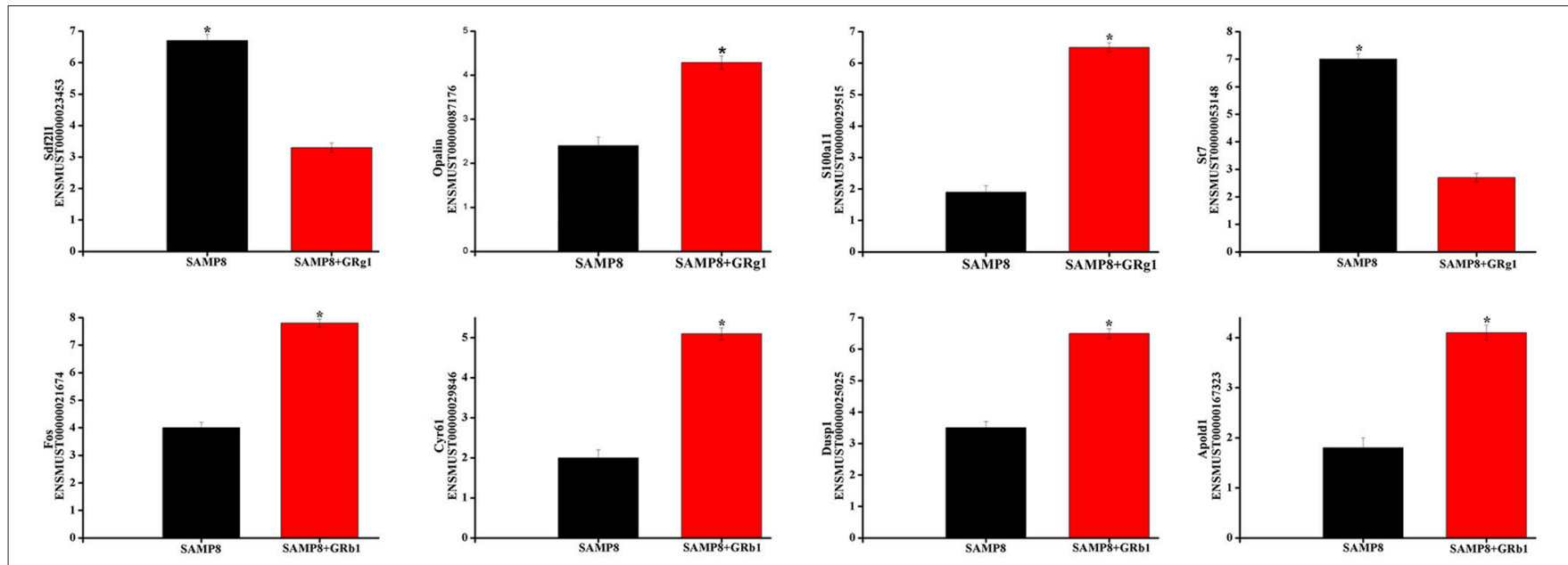

FIGURE 4 | Validation of transcript expression by qPCR. Data are presented as the mean \pm SE $(n=3)$. $\beta$-actin gene was used as a housekeeping internal control. Transcript expression was quantified relative to the expression level of $\beta$-actin using the comparative cycle threshold $(\Delta \mathrm{CT})$ method. ${ }^{*} p<0.05$.

TABLE 1 | AD-related genes identified after treatment with GRg1.

\begin{tabular}{llccr}
\hline Gene & Transcript ID & $\begin{array}{c}\text { SAMP8 } \\
\text { FPKM }\end{array}$ & $\begin{array}{c}\text { SAMP8+Rg1 } \\
\text { FPKM }\end{array}$ & $\begin{array}{r}\text { SAMR1 } \\
\text { FPKM }\end{array}$ \\
\hline Bche & ENSMUST00000029367 & 0.98 & 1.33 & 1.30 \\
Fgfr1 & ENSMUST00000084027 & 1.37 & 2.21 & 2.23 \\
Dusp1 & ENSMUST00000025025 & 9.07 & 10.89 & 16.53 \\
Cdkn2b & ENSMUST00000097981 & 0.29 & 0.52 & 0.62 \\
Flna & ENSMUST00000114299 & 3.23 & 4.64 & 4.87 \\
Glp1r & ENSMUST00000114574 & 0.27 & 0.48 & 0.77 \\
Slc39a7 & ENSMUST00000169397 & 5.97 & 1.98 & 2.59 \\
Calr & ENSMUST00000003912 & 196.95 & 159.62 & 163.60 \\
Mapk11 & ENSMUST00000088823 & 14.36 & 12.77 & 11.81 \\
Trpc6 & ENSMUST00000050433 & 22.02 & 18.76 & 4.37 \\
Smad3 & ENSMUST00000034973 & 20.09 & 16.76 & 16.99 \\
Pdia6 & ENSMUST00000057288 & 85.99 & 61.29 & 68.01 \\
Pdia4 & ENSMUST00000077290 & 36.14 & 24.39 & 29.83 \\
Xbp1 & ENSMUST00000063084 & 55.75 & 39.55 & 42.90 \\
Arntl & ENSMUST00000047321 & 17.87 & 13.26 & 13.49 \\
Gm10053 & ENSMUST00000073080 & 1.79 & 1.06 & 0.42 \\
Pfkfb3 & ENSMUST00000028114 & 5.22 & 3.46 & 4.07 \\
Htr5b & ENSMUST00000055884 & 0.85 & 0.47 & 0.48 \\
\hline & & & & \\
\hline
\end{tabular}

FPKM, fragments per kilobase of exon per million fragments mapped. FPKM was used to determine the differential expression of the transcripts.

found in the SAMP8 vs. SAMP8+GRb1 group. Subsequently, qPCR was used to measure the accuracy of RNA sequencing. Eight dysregulated mRNAs were randomly selected for the test. All results were consistent with the RNA-seq data. The qPCR results confirmed that our resultant mRNAs were of high quality. Evidently, this research provides a solid foundation for further exploration.

GO and KEGG pathway analyses were also performed on the coding genes associated with the significant mRNAs to study the possible biological, cellular, and molecular functions of
TABLE 2 | AD-related genes identified after treatment with GRb1.

\begin{tabular}{llccr}
\hline Gene & Transcript ID & $\begin{array}{c}\text { SAMP8 } \\
\text { FPKM }\end{array}$ & $\begin{array}{c}\text { SAMP8+Rb1 } \\
\text { FPKM }\end{array}$ & $\begin{array}{r}\text { SAMR1 } \\
\text { FPKM }\end{array}$ \\
\hline Bche & ENSMUST00000029367 & 0.98 & 1.27 & 1.30 \\
Kat2a & ENSMUST00000103118 & 1.24 & 2.24 & 2.50 \\
Dusp1 & ENSMUST00000025025 & 9.07 & 16.56 & 16.53 \\
Cdkn2b & ENSMUST00000097981 & 0.29 & 0.54 & 0.62 \\
Fos & ENSMUST00000021674 & 11.40 & 20.61 & 17.49 \\
Glp1r & ENSMUST00000114574 & 0.27 & 0.48 & 0.77 \\
Nr4a1 & ENSMUST00000023779 & 17.55 & 27.11 & 25.48 \\
Calr & ENSMUST00000003912 & 196.95 & 171.07 & 163.60 \\
Mapk11 & ENSMUST00000088823 & 14.36 & 11.96 & 11.81 \\
Mme & ENSMUST00000029400 & 0.80 & 1.48 & 1.62 \\
Smad3 & ENSMUST00000034973 & 20.09 & 16.09 & 16.99 \\
Pdia6 & ENSMUST00000057288 & 85.99 & 67.21 & 68.01 \\
Pdia4 & ENSMUST00000077290 & 36.14 & 26.71 & 29.83 \\
Xbp1 & ENSMUST00000063084 & 55.75 & 43.00 & 42.90 \\
Syk & ENSMUST00000055087 & 0.73 & 0.33 & 0.53 \\
Htr3a & ENSMUST00000003826 & 4.05 & 3.33 & 3.20 \\
Pfkfb3 & ENSMUST00000028114 & 5.22 & 3.19 & 4.07 \\
Htr5b & ENSMUST00000055884 & 0.85 & 0.46 & 0.48 \\
\hline
\end{tabular}

FPKM, fragments per kilobase of exon per million fragments mapped. FPKM was used to determine the differential expression of the transcripts.

these mRNAs in $\mathrm{AD}$ progression. $\mathrm{AD}$ is a multifactorial disease, and many theories have been advanced concerning its causes, including neuron loss, $\mathrm{A} \beta$ deposition, tau neuropathology, immune system dysfunction, synapse injury, oxidative stress, and mitochondrial dysfunction (Armstrong, 2013). As expected, our analysis showed that GRg1 and GRb1 exert multi-level anti-AD effects; for example, the MAPK signaling pathway, Notch signaling pathway, PI3K-Akt signaling pathway, and GO terms (e.g., GO: 0000188, GO: 0007420, and GO: 0031175) were affected. 
To conceptualize these findings, we established reasonable limitations and further refined the closest results. After treatment with GRg1, we identified 6 upregulated genes, namely, Bche, Fgfr1, Dusp1, Cdkn2b, Flna, and Glp1r, and 12 downregulated genes, namely, Slc39a7, Calr, Mapk11, Trpc6, Smad3, Pdia6, Pdia4, Xbp1, Arntl, Gm10053, Pfkfb3, and Htr5b. Meanwhile, in the GRb1 experimental group, 8 genes (Bche, Kat2a, Dusp1, Cdkn2b, Fos, Nr4a1, Mme, and Glp1r) were up-regulated, and 10 genes (Calr, Mapk11, Smad3, Pdia6, Pdia4, Xbp1, Pfkfb3, Syk, $H t r 3 a$, and $H t r 5 b$ ) were downregulated. Interestingly, 12 genes, including 4 upregulated genes (Bche, Dusp1, Cdkn2b, and Glp1r) and 8 downregulated genes (Calr, Mapk11, Smad3, Pdia6, Pdia4, Xbp1, Pfkfb3, and Htr5b) were affected by both GRg1 and GRb1. All of the abovementioned genes showed an expression similar to that in the normal mice (SAMR1). In addition, these genes are highly relevant to AD. Earlier in the article, we discussed Mapk11 (Stein et al., 1997). Neprilysin, which is encoded by Mme, is one of the most important $A \beta$-degrading enzymes in the prevention of AD pathology (Miners et al., 2012). Trpc6, a transient receptor potential canonical (TRPC) related gene, has been reported as the molecular entity associated with $\mathrm{Ca}^{2+}$ entry activity (Zhu et al., 1996) and may participate in the AD process. Brain development is impaired in fos -/- mice (Velazquez et al., 2015). As observed, GRg1 and GRb1 are effective Chinese medicines that can be used to prevent and treat $\mathrm{AD}$ with multi-system, multi-target, and multi-directional comprehensive regulation. However, assessment of the curative effects of GRg1 and GRb1 merits further investigation in large-scale, clinical studies. This endeavor is going to be an enormous challenge in the future.

\section{REFERENCES}

Alzheimer's Association. (2015). 2015 Alzheimer's disease facts and figures. Alzheimers. Dement. 11, 332-384. doi: 10.1016/j.jalz.2015.02.003

Armstrong, R. A. (2013). What causes Alzheimer's disease? Folia. Neuropathol. 51, 169-188. doi: 10.5114/fn.2013.37702

Babusikova, E., Evinova, A., Dobrota, D., Jurecekova, J., and Jesenak, M. (2011). Alzheimer's Disease: Definition, Molecular and Genetic Factors. Rijeka: INTECH Open Access Publisher.

Bhat, N. R. (2010). Linking cardiometabolic disorders to sporadic Alzheimer's disease: a perspective on potential mechanisms and mediators. J. Neurochem. 115, 551-562. doi: 10.1111/j.1471-4159.2010.06978.x

Butterfield, D. A., and Poon, H. F. (2005). The senescence-accelerated prone mouse (SAMP8): a model of age-related cognitive decline with relevance to alterations of the gene expression and protein abnormalities in Alzheimer's disease. Exp. Gerontol. 40, 774-783. doi: 10.1016/j.exger.2005.05.007

Cheng, X. R., Zhou, W. X., and Zhang, Y. X. (2014). The behavioral, pathological and therapeutic features of the senescence-accelerated mouse prone 8 strain as an Alzheimer's disease animal model. Ageing Res. Rev. 13, 13-37. doi: 10.1016/j.arr.2013.10.002

Clark, J. D., Gebhart, G. F., Gonder, J. C., Keeling, M. E., and Kohn, D. F. (1997). Special report: the 1996 guide for the care and use of laboratory Animals. ILAR J. 38, 41-48. doi: 10.1093/ilar.38.1.41

D'Hooge, R., and De Deyn, P. P. (2001). Applications of the Morris water maze in the study of learning and memory. Brain Res. Rev. 36, 60-90. doi: 10.1016/S0165-0173(01)00067-4

Du, Q., Jerz, G., Waibel, R., and Winterhalter, P. (2003). Isolation of dammarane saponins from Panax notoginseng by high-speed counter-current chromatography. J. Chromatogr. A 1008, 173-180. doi: 10.1016/S0021-9673(03)00988-9
In conclusion, we investigated SAMP8 mice brain mRNAs after 4 months of GRg1 and GRb1 administration. Evidently, intake of GRg1 and GRb1 restored the cognitive function of SAMP8 mouse and exerted robust neuroprotective effects in different ways. This strategy provides an invaluable resource for the clinical application of GRg1 and GRb1 in treating AD.

\section{AUTHOR CONTRIBUTIONS}

Wrote the paper, Conceived and designed the experiments: SZ. Performed the experiments: SZ, DZ and WZ. Analyzed the data: SZ, DZ, HL, HZ, CF and WZ. Contributed reagents/materials/analysis tools: $\mathrm{SZ}$ and $\mathrm{WZ}$.

\section{ACKNOWLEDGMENTS}

We thank all the contributors of this study. This work was supported by grants from the National Natural Science Foundation of China (No. 81274118), the Key New Drug Creation and Development Program of China (No. 2012ZX09103-201), and the Fundamental Research Funds for Central Universities.

\section{SUPPLEMENTARY MATERIAL}

The Supplementary Material for this article can be found online at: http://journal.frontiersin.org/article/10.3389/fphar. 2017.00088/full\#supplementary-material

Howes, M. J., Perry, N. S., and Houghton, P. J. (2003). Plants with traditional uses and activities, relevant to the management of Alzheimer's disease and other cognitive disorders. Phytother. Res. 17, 1-18. doi: 10.1002/ptr.1280

Kang, L., Li, S., Xing, Z. G., Li, J. Z., Su, Y. H., Fan, P., et al. (2014). Dihydrotestosterone treatment delays the conversion from mild cognitive impairment to Alzheimer's disease in SAMP8 mice. Horm. Behav. 65, 505-515. doi: 10.1016/j.yhbeh.2014.03.017

Kim, D., Pertea, G., Trapnell, C., Pimentel, H., Kelley, R., and Salzberg, S. L. (2013). TopHat2: accurate alignment of transcriptomes in the presence of insertions, deletions and gene fusions. Genome. Biol. 14:R36. doi: 10.1186/gb-2013-14-4-r36

Kim, M. H., Kim, S. H., and Yang, W. M. (2014). Mechanisms of action of phytochemicals from medicinal herbs in the treatment of Alzheimer's disease. Planta Med. 80, 1249-1258. doi: 10.1055/s-0034-1383038

Li, H., Deng, C. Q., Chen, B. Y., Zhang, S. P., Liang, Y., and Luo, X. G. (2009). Total saponins of Panax notoginseng modulate the expression of caspases and attenuate apoptosis in rats following focal cerebral ischemia-reperfusion. $J$. Ethnopharmacol. 121, 412-418. doi: 10.1016/j.jep.2008.10.042

Li, N., Zhou, L., Li, W., Liu, Y., Wang, J., and He, P. (2015). Protective effects of ginsenosides $\mathrm{Rg} 1$ and $\mathrm{Rb} 1$ on an Alzheimer's disease mouse model: a metabolomics study. J. Chromatogr. B Analyt. Technol. Biomed. Life. Sci. 985, 54-61. doi: 10.1016/j.jchromb.2015.01.016

Ma, Q., Qiang, J., Gu, P., Wang, Y., Geng, Y., and Wang, M. (2011). Agerelated autophagy alterations in the brain of senescence accelerated mouse prone 8 (SAMP8) mice. Exp. Gerontol. 46, 533-541. doi: 10.1016/j.exger.2011. 02.006

Mao, X., Cai, T., Olyarchuk, J. G., and Wei, L. (2005). Automated genome annotation and pathway identification using the KEGG Orthology $(\mathrm{KO})$ as a controlled vocabulary. Bioinformatics 21, 3787-3793. doi: 10.1093/bioinformatics/bti430 
Miners, S., van Helmond, Z., Barker, R., Passmore, P. A., Johnston, J. A., Todd, S., et al. (2012). Genetic variation in MME in relation to neprilysin protein and enzyme activity, A $\beta$ levels, and Alzheimer's disease risk. Int. J. Mol. Epidemiol. Genet. 3, 30-38.

Ozsolak, F., and Milos, P. M. (2011). RNA sequencing: advances, challenges and opportunities. Nat. Rev. Genet. 12, 87-98. doi: 10.1038/nrg2934

Roy, D. S., Arons, A., Mitchell, T. I., Pignatelli, M., Ryan, T. J., and Tonegawa, S. (2016). Memory retrieval by activating engram cells in mouse models of early Alzheimer's disease. Nature 531, 508-512. doi: 10.1038/nature 17172

Stein, B., Yang, M. X., Young, D. B., Janknecht, R., Hunter, T., Murray, B. W., et al. (1997). p38-2, a novel mitogen-activated protein kinase with distinct properties. J. Biol. Chem. 272, 19509-19517. doi: 10.1074/jbc.272.31.19509

Trapnell, C., Pachter, L., and Salzberg, S. L. (2009). TopHat: discovering splice junctions with RNA-Seq. Bioinformatics 25, 1105-1111. doi: 10.1093/bioinformatics/btp120

Trapnell, C., Williams, B. A., Pertea, G., Mortazavi, A., Kwan, G., van Baren, M. J., et al. (2010). Transcript assembly and quantification by RNA-Seq reveals unannotated transcripts and isoform switching during cell differentiation. Nat. Biotechnol. 28, 511-515. doi: 10.1038/nbt.1621

Velazquez, F. N., Prucca, C. G., Etienne, O., D’Astolfo, D. S., Silvestre, D. C., Boussin, F. D., et al. (2015). Brain development is impaired in c-fos -/- mice. Oncotarget 6, 16883-16901. doi: 10.18632/oncotarget.4527

Vorhees, C. V., and Williams, M. T. (2006). Morris water maze: procedures for assessing spatial and related forms of learning and memory. Nat. Protoc. 1, 848-858. doi: 10.1038/nprot.2006.116

Wang, Y., Liu, J., Zhang, Z., Bi, P., Qi, Z., and Zhang, C. (2011). Anti-neuroinflammation effect of ginsenoside $\mathrm{Rbl}$ in a rat model of Alzheimer disease. Neurosci. Lett. 487, 70-72. doi: 10.1016/j.neulet.2010. 09.076

Yaari, R., and Corey, B. J. (2007). Alzheimer's disease. Semin. Neurol. 27, 32-41. doi: $10.1055 /$ s-2006-956753
Yan, S., Li, Z., Li, H., Arancio, O., and Zhang, W. (2014). Notoginsenoside R1 increases neuronal excitability and ameliorates synaptic and memory dysfunction following amyloid elevation. Sci. Rep. 4:6352. doi: 10.1038/srep06352

Young, M. D., Wakefield, M. J., Smyth, G. K., and Oshlack, A. (2010). Gene ontology analysis for RNA-seq: accounting for selection bias. Genome Biol. 11:R14. doi: 10.1186/gb-2010-11-2-r14

Zhang, J., and Fang, X. Y. (2004). The historical condition in the spread of Sanqi (Panax notoginseng) in the Ming Dynasty. Zhonghua Yi Shi ZA Zhi 34, 16-20. doi: 10.3760/cma.j.issn.0255-7053.2004.01.003

Zhang, S., Qin, C., Cao, G., Xin, W., Feng, C., and Zhang, W. (2016). Systematic analysis of long noncoding RNAs in the senescence-accelerated mouse prone 8 brain using RNA sequencing. Mol. Ther. Nucleic Acids 5:e343. doi: $10.1038 / \mathrm{mtna} .2016 .57$

Zhao, B. S., Liu, Y., Gao, X. Y., Zhai, H. Q., Guo, J. Y., and Wang, X. Y. (2014). Effects of ginsenoside Rg1 on the expression of toll-like receptor 3, 4 and their signalling transduction factors in the NG108-15 murine neuroglial cell line. Molecules 19, 16925-16936. doi: 10.3390/molecules191016925

Zhu, X., Jiang, M., Peyton, M., Boulay, G., Hurst, R., Stefani, E., et al. (1996). Trp, a novel mammalian gene family essential for agonist-activated capacitative $\mathrm{Ca}^{2+}$ entry. Cell 85, 661-671. doi: 10.1016/S0092-8674(00)81233-7

Conflict of Interest Statement: The authors declare that the research was conducted in the absence of any commercial or financial relationships that could be construed as a potential conflict of interest.

Copyright (c) 2017 Zhang, Zhu, Li, Zhang, Feng and Zhang. This is an open-access article distributed under the terms of the Creative Commons Attribution License (CC $B Y)$. The use, distribution or reproduction in other forums is permitted, provided the original author(s) or licensor are credited and that the original publication in this journal is cited, in accordance with accepted academic practice. No use, distribution or reproduction is permitted which does not comply with these terms. 